\title{
Protein-linked glycans in periodontal bacteria: prevalence and role at the immune interface
}

\author{
Rajendra P. Settem ${ }^{1}$, Kiyonobu Honma ${ }^{1}$, Graham P. Stafford ${ }^{2}$ and Ashu Sharma ${ }^{1}$ * \\ ${ }^{1}$ Department of Oral Biology, School of Dental Medicine, University at Buffalo, State University of New York, Buffalo, NY, USA \\ ${ }^{2}$ Oral and Maxillofacial Pathology, School of Clinical Dentistry, Claremont Crescent, University of Sheffield, Sheffield, UK
}

\section{Edited by:}

Amy Rasley, Lawrence Livermore

National Laboratory, USA

\section{Reviewed by:}

Dina Weilhammer, Lawrence

Livermore National Laboratory, USA

June Round, University of Utah, USA

${ }^{*}$ Correspondence:

Ashu Sharma, Department of Oral Biology, School of Dental Medicine, University at Buffalo, State University of New York, 311 Foster Hall, 3435

Main Street, Buffalo, NY 14214, USA e-mail:sharmaa@buffalo.edu
Protein modification with complex glycans is increasingly being recognized in many pathogenic and non-pathogenic bacteria, and is now thought to be central to the successful life-style of those species in their respective hosts. This review aims to convey current knowledge on the extent of protein glycosylation in periodontal pathogenic bacteria and its role in the modulation of the host immune responses. The available data show that surface glycans of periodontal bacteria orchestrate dendritic cell cytokine responses to drive $T$ cell immunity in ways that facilitate bacterial persistence in the host and induce periodontal inflammation. In addition, surface glycans may help certain periodontal bacteria protect against serum complement attack or help them escape immune detection through glycomimicry. In this review we will focus mainly on the generalized surface-layer protein glycosylation system of the periodontal pathogen Tannerella forsythia in shaping innate and adaptive host immunity in the context of periodontal disease. In addition, we will also review the current state of knowledge of surface protein glycosylation and its potential for immune modulation in other periodontal pathogens.

Keywords: protein glycosylation, periodontal bacteria, immune response

\section{INTRODUCTION}

Until relatively recently, protein glycosylation was believed to be restricted only to eukaryotic proteins. However, with the availability of genome-scale information and sophisticated mass spectrometry as well as nuclear magnetic resonance techniques primarily from the past decade it has come to light that protein glycosylation occurs quite frequently in bacteria as well (Nothaft and Szymanski, 2010). More and more bacterial proteins of Gram-positive and Gram-negative origin are frequently being identified with $\mathrm{N}$ - and $\mathrm{O}$-linked glycan modifications (Ashline etal., 2005; Brisson etal., 2010; reviewed in Iwashkiw etal., 2012, 2013). Moreover, it is becoming apparent that protein glycosylation is also prevalent amongst Archaea (Eichler, 2013; Kandiba and Eichler, 2013). Dedicated computational algorithms have also been developed to predict glycosylated residues in prokaryotic proteins (Chauhan etal., 2012), and a web program, GlycoPP at http://www.imtech.res.in/raghava/glycopp/index.html, using such algorithms is currently available for predicting $N$ - and $O$-glycosylation sites in prokaryotic protein sequences with an accuracy of $70-80 \%$. In most cases bacterial glycans are linked directly to asparagine or serine/threonine residues within specific peptide motifs for $\mathrm{N}$ - and $\mathrm{O}$-glycosylation, respectively. Proteins such as flagellins, pilins, and surface (S)-layer proteins have been shown to be glycosylated quite extensively in bacteria by dedicated glycosylation systems (Nothaft and Szymanski, 2010). Recent studies have alluded to the role of protein glycosylation in immune modulation in pathogenic as well as commensal bacteria, such as in Neisseria species (Vik et al., 2009), Campylobacter jejuni (Szymanski and Wren, 2005), and Bacteroides fragilis (Fletcher etal., 2009), and in attachment and infective ability of Chlamydia trachomatis (Kuo et al., 1996) and C. jejuni species (Szymanski et al., 2002; Karlyshev et al., 2004). In addition, variations in flagellar protein-linked glycans in C. jejuni have been shown to cause phenotypic phasevariations and antigenic diversity, important for the pathogen's virulence (Guerry et al., 2006; Young et al., 2007; Hitchen et al., 2010).

With respect to the human oral cavity, the niche is colonized with nearly 700 bacterial species with different taxa and phylotypes (Dewhirst et al., 2010). Of these diverse bacteria, a select group of Gram-negative anaerobes residing in the subgingival crevices (spaces between the teeth and gums; Figure 1) as biofilms induce one of the most common forms of the inflammatory disease in humans, known as periodontitis. This disease is characterized by the progressive destruction of the tooth supporting apparatus and often leads to tooth loss in adults. In addition, chronic infections with periodontal pathogens have been linked to the risk for systemic health conditions such as cardiovascular, diabetic, respiratory, and arthritic diseases (Beck et al., 2008; Genco, 2009; Gomes-Filho et al., 2009; Genco and Van Dyke, 2010; Scher etal., 2012). A group of bacteria known as the "red complex" comprising Porphyromonas gingivalis, Treponema denticola, and Tannerella forsythia has been strongly implicated in the initiation and progression of periodontitis (Socransky et al., 1998). We discuss below recent data mainly from studies in $T$. forsythia and $P$. gingivalis that suggest these bacteria utilize protein glycosylation for undermining the host immunity to persist in the host and cause periodontal destruction. 


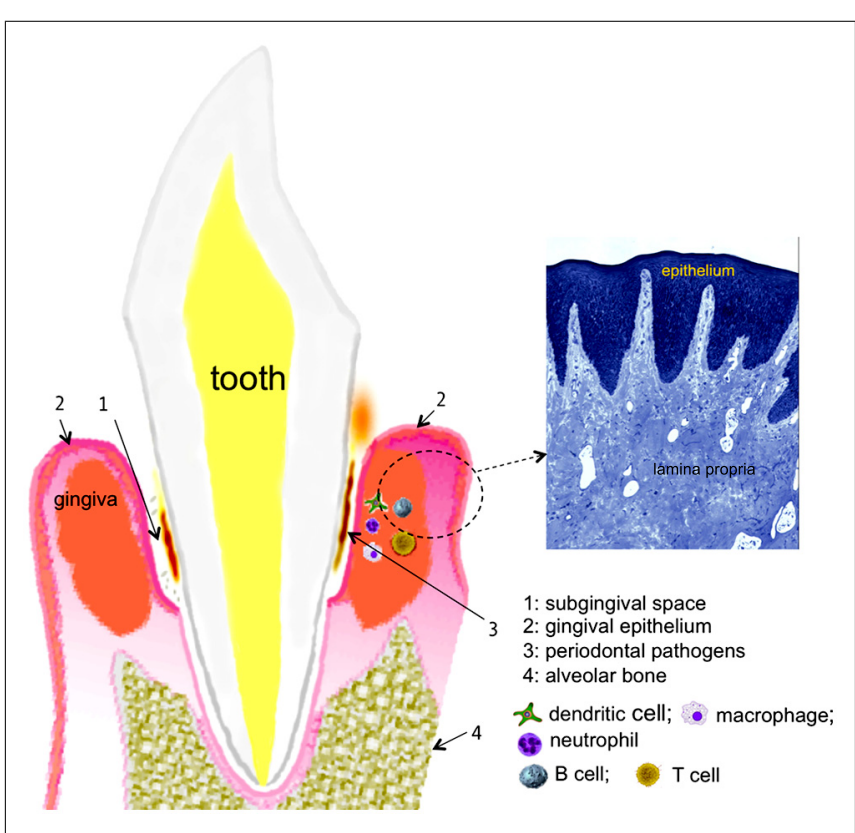

FIGURE 1 | Schematic representation of the periodontal tissues supporting the tooth. A histological section of the gingival tissue stained with toluidine blue is shown on the right (courtesy, Dr. Moon-ll Cho, University at Buffalo).

\section{PROTEIN-LINKED GLYCANS OF PERIODONTAL BACTERIA AS IMMUNOMODULATORS}

The periodontal pathogen $T$. forsythia possesses a S-layer, a toothlike serrated layer covering the outer-membrane (Sabet et al., 2003; Figure 2A). S-layers are comprised of water insoluble proteins intrinsically capable of self-assembling into crystalline lattice and are believed to provide protection against the deleterious effects of the environment as well host immune components (Sara and Sleytr, 2000). S-layer proteins in Gram-positive bacteria may be glycosylated by the addition of O-linked long-chain glycan repeats in a process similar to lipopolysaccharide (LPS) biosynthesis (Messner et al., 2008). The T. forsythia S-layer, however, is unique in that it is the only Gram-negative S-layer known to date which is glycosylated (Lee etal., 2006). It is comprised of two high molecular weight glycoproteins, TfsA $(200 \mathrm{kDa})$ and TfsB $(210 \mathrm{kDa})$ which are clearly prominent in the protein profiles of this organism and make up a large proportion of the total bacterial protein.

Our previous work and that of the Schaffer group (using our wecC mutant) showed that a putative exopolysaccharide synthesis operon wecC-neuC-gtf is involved in the O-glycosylation of Tannerella S-layer and other surface proteins (Honma et al., 2007; Posch etal., 2011). Specifically, the wecC gene product (UDP-N-acetylmannosaminuronic acid dehydrogenase homolog) was shown to be involved in the addition of a three-sugarbranch motif comprising two sub-terminal mannosuronic acid (ManNAcA) and a terminal modified pseudaminic acid residues (Pse5Am7Gc, 5-acetimidol-7- $N$-glycolyl-pseudaminic acid) on an $O$-linked oligosaccharide core attached to the surface glycoproteins (Posch et al., 2011; Figure 2B). The glycan core is attached to the S-layer protein (and several other Tannerella surface proteins) at several sites in each protein via $\mathrm{O}$-linkages at Ser, Thr, or Tyr residues within the three amino acid motif $\mathrm{D}(\mathrm{S}, \mathrm{T})(\mathrm{A}, \mathrm{L}, \mathrm{V}, \mathrm{I}, \mathrm{M}, \mathrm{T})$ (Posch et al., 2011). This mode of glycosylation at specific three amino acid motifs appears to be well conserved across a range of Bacteroidetes species. This is evidenced by elegant transcomplementation experiments in which the T. forsythia S-layer protein was produced and glycosylated at this motif with the $B$. fragilis specific glycan, and vice versa, i.e., a $B$. fragilis protein was glycosylated in T. forsythia with the Tannerella glycan (Posch et al., 2013). In fact this has now been shown to be the case in a range of Bacteroides, Prevotella, and Porphyromonas species with bioinformatic (Fletcher et al., 2011) as well as experimental approaches using an antibody against the core component of the Bacteroides group O-glycan (Coyne et al., 2013). This conservation of a general $\mathrm{O}$-glycosylation system in Bacteroidetes is indicative of its potentially essential function in divergent Bacteroidetes species living in wide-ranging niches.

Previous studies have shown that the Tannerella S-layer is capable of mediating bacterial attachment to and invasion of epithelial cells (Lee et al., 2006). Furthermore, for the benefit of the bacterium, the S-layer helps to blocks the early release of inflammatory cytokines by immune cells. The role of S-layer glycosylation on the bacterium's ability to directly modulate host immunity to impact its in vivo life-cycle has now come to light. A recent study from our laboratory showed that loss of the terminal trisaccharide branch motif on the S-layer as well as a number of other $\mathrm{O}$-glycosylated surface proteins due to wecC deficiency renders the bacterium increasingly prone to recognition and processing by antigen-presenting cells (Settem et al., 2012). Specifically, the loss of this terminal tri-saccharide branch (Figure 2B) promotes phagocytosis of the bacterium by a subset of antigen presenting cells called dendritic cells (DCs) and concurrent induction of Th17-activating cytokines IL-6 and IL-23 (Settem et al., 2012). This modulation of DC responses has major consequences for pathogenesis since DCs reside as immune system sentinels within tissues that constantly encounter invading microbes. In tissues they recognize different pathogen-associated molecular patterns (PAMPs) and act to direct innate and adaptive immune responses locally via pathogen-recognition receptors (PRRs), a group of molecules which include Toll-like receptors (TLRs), nucleotide oligomerization domain receptors (NODs), and C-type lectin-like receptors (CLRs; Takeuchi and Akira, 2010). It is the latter of these, the CLRs, which are most pertinent to the recognition of bacterial $O$-glycans since their function is to act as carbohydrate binding receptors. Thus, surface $O$ linked glycans, the tri-saccharide motif in particular, impact DC-mediated processing of the bacterium, also impacting the activation of intracellular receptors and inflammasome processing for IL-23 induction. This manipulation of DC behavior via carbohydrate ligands is a growing theme in bacterial pathogen biology and several recent studies indicate that bacterial polysaccharides can block recognition and phagocytosis of microbes by DCs and macrophages (Meijerink et al., 2012). Moreover, microbeassociated glycans regulate DC maturation and cytokine secretion to affect polarization of naïve $\mathrm{T}$ cells into specific subtypes upon microbial infection, with signaling crosstalk between CLRs and 

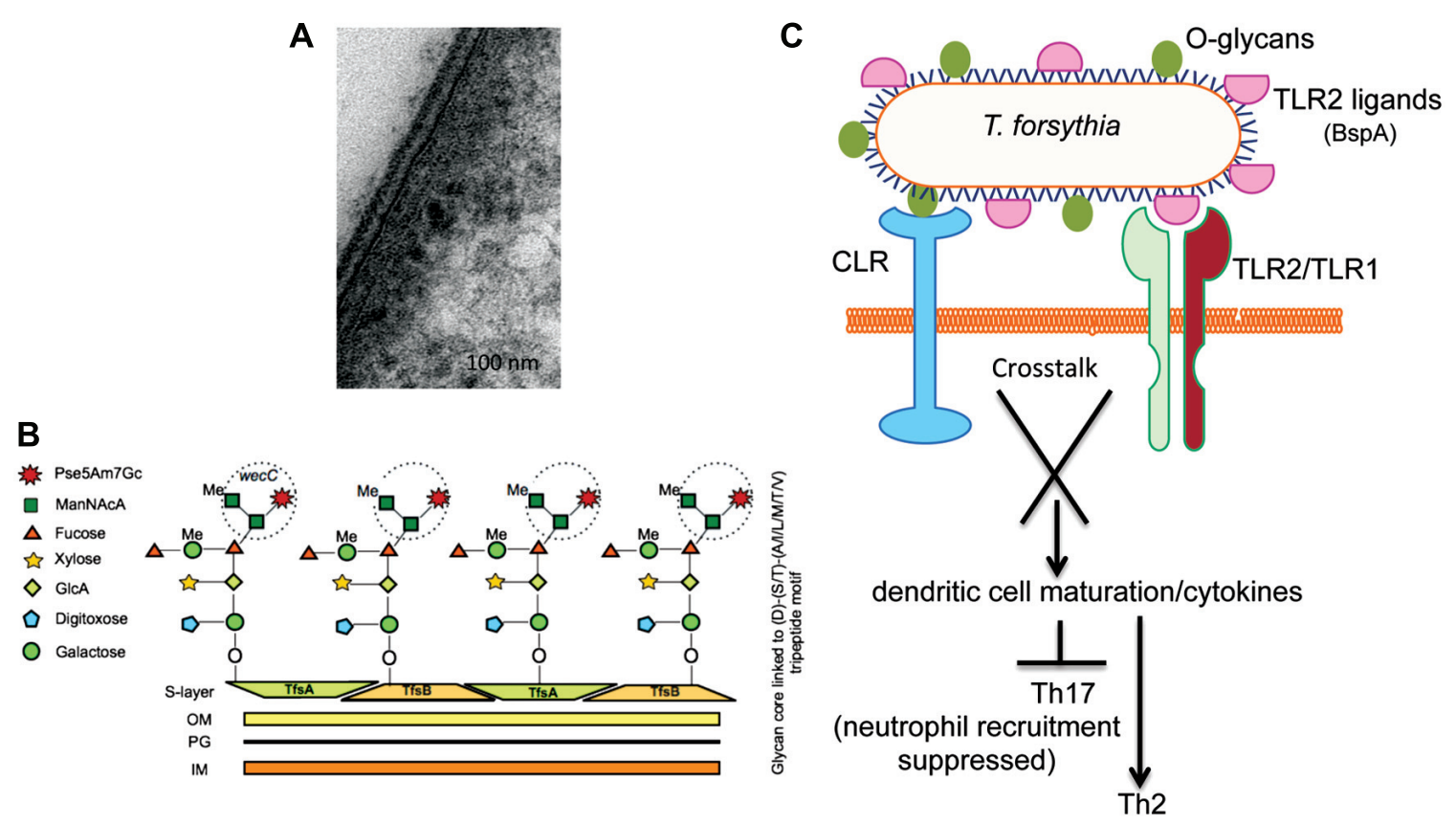

FIGURE 2 | (A) Transmission electron microscopy (TEM) micrographs of $T$. forsythia ultrathin sections. (B) Schematic diagram of the O-glycan core structure linked to a peptide motif in Tannerella surface-layer proteins. A terminal trisaccharide motif synthesized by the wecC operon is circled. (C) Signaling crosstalk between a C-type lectin-like receptor (CLR) and TLR2 activated by O-glycans and TLR2 ligands (e.g., BspA), respectively, may orchestrate $T$ helper cell differentiation.
TLRs thought to influence this development (Geijtenbeek and Gringhuis, 2009). In the case of T. forsythia, O-glycan-mediated signaling via a CLR might synergize with TLR2-dependent signaling induced by the bacterium's major virulence factor and TLR2 ligand, the BspA protein (Onishi et al., 2008; Myneni et al., 2012; Figure 2C).

Indeed, in line with the above predictions, the terminal $O$ glycan motif attached to the surface proteins of $T$. forsythia does seem to be a key factor in influencing $\mathrm{T}$ cell polarization during T. forsythia infection. The evidence to support this comes from data generated in mouse models infected with wild-type bacteria (decorated with the $O$-glycan) which induce robust TLR2dependent Th2-biased inflammatory responses, leading to alveolar bone resorption (Myneni et al., 2011). Strikingly, this TLR2-Th2 bias is perturbed in response to infection with a bacterial strain lacking the terminal tri-saccharide $O$-glycan (i.e., a wecC mutant, Figure 2B) and when mice are infected with the strain lacking this terminal three-sugar branch motif on the $O$-linked glycan core, T cells polarize into the Th17 subset (Settem et al., 2012). This leads to increased infiltration of neutrophils into the gingival tissues and therefore decreased persistence of bacteria in periodontal tissues, resulting in reduced alveolar bone loss compared to the mice infected with the wild-type strain (Settem et al., 2012). Taken together, these data suggest that the surface $O$-glycan motif of T. forsythia is involved in suppressing Th17 responses, which allow increased bacterial persistence, promoting Th2-dependent inflammatory alveolar bone destruction (Figure 2C).

The other "red complex" periodontal pathogen P. gingivalis possesses two types of cell-surface fimbriae, the major fimbria known as FimA and the minor fimbria known as Mfal. Mfal is comprised of a $67-\mathrm{kDa}$ protein with $\mathrm{N}$ - and $\mathrm{O}$-linked fucose, mannose, $N$-acetylglucosamine, and $N$-acetylgalactosamine sugars (Zeituni et al., 2010). Mfal activates DC-SIGN (dendritic cell-specific intercellular adhesion molecule-3-grabbing non-integrin) lectin on DCs. On the other hand, the 43-kDa major fimbrial protein FimA is non-glycosylated and activates TLR2. Recent studies further suggest that a crosstalk between TLR2 and DC-SIGN signaling due to FimA and Mfal fimbria, respectively, modulates the expression of Th cell differentiation cytokines in DCs against $P$. gingivalis, and is thus thought to orchestrate specific $\mathrm{T}$ cell immunity (Zeituni et al., 2010).

Orchestration of Th bias through the mediation of glycan modifications is not confined to periodontal pathogens alone, but is widely observed in the gastrointestinal pathogens and commensals. In the case of C. jejuni, surface capsular polysaccharide modifications with methyl phosphoramidate have been shown to modulate Th17 immunity against the pathogen and thus impact bacterial persistence in the host (Maue et al., 2012). With respect to the ubiquitous commensal and opportunistic pathogen $-B$. fragilis, the expression of its polysaccharide antigen (PSA) helps to keep inflammatory $\mathrm{T}$ cell lineages (e.g., Th17) in check and simultaneously promote IL-10-producing FoxP3(+) Treg cells in the gut (Mazmanian et al., 2008; Ochoa-Reparaz et al., 2010; Surana and Kasper, 2012). This role of PSA in influencing T cell populations is so profound that PSA-deficient bacteria skew the balance so far toward Th17 cells that infected mice develop colitis. Similar types of zwitterionic polysaccharides (ZPS) with immunomodulatory roles have also been identified in bacteria 
such as Streptococcus pneumoniae and Staphylococcus aureus type5 and -8 (Tzianabos et al., 2000; Gonzalez-Fernandez et al., 2008). A clear mechanistic understanding of how bacterial polysaccharide regulate $\mathrm{T}$ cell polarization is lacking in light of the fact that PSAs are generally not presented to $\mathrm{T}$ cells ( $\mathrm{T}$ cell independent) via antigen presenting cells. Rather, it has been demonstrated that ZPS antigens can be presented to T cells by APCs in an major histocompatibility complex class II (MHC-II) restricted manner (Avci and Kasper, 2010). This picture is further complicated by evidence suggesting that many oligosaccharides linked to peptides can directly activate T cells (Avci et al., 2013). Whether or not Tannerella polysaccharides are recognized by MHC-II or directly activate $\mathrm{T}$ cells is currently unknown. Alternatively, modulation of DCs through activation of CLRs such as DC-SIGN by Tannerella glycans could plausibly regulate the secretion of $\mathrm{T}$ cell biasing cytokines. In support of this notion, DC-SIGN is known to exhibit crosstalk with TLRs and impact the maturation of DCs and differentially regulate the cytokine environment, promoting polarization of $\mathrm{T}$ cells into specific subsets (Geijtenbeek and Gringhuis, 2009; Geijtenbeek et al., 2009; van den Berg et al., 2012).

\section{OTHER GLYCAN-RELATED FACTORS OF PERIODONTAL BACTERIA}

It is also worth noting that several glycoproteins with potential roles in bacterial pathogenicity, including the arginine- (RgpB and RgpA) and lysine-specific (Kgp) cysteine proteinases (collectively known as gingipains) and major outer-membrane protein pgm6 (OmpA) have been reported in $P$. gingivalis (Kishi et al., 2012). Gingipain proteases are major virulence factors of $P$. gingivalis that paralyze host immune defenses by degrading serum complement, antibodies and chemokines, and $\mathrm{T}$ cell biasing cytokines (Guo et al., 2010). Although glycans linked to gingipains per se do not play any direct role in corrupting host defense molecules, post-translation polysaccharide modifications via a novel protein secretion system (PorSS; Paramonov et al., 2005; Glew et al., 2012) as well as a Vim (virulence modulating) dependent glycosylation system (Aruni et al., 2013) play an essential role in the biogenesis of these proteases in P. gingivalis. In addition, recent work of Comstock and colleagues on the general $O$-glycosylation system in Bacteroidales species suggests the presence of a shared $O$-glycosylation system in a range of periodontal pathogens including $P$. gingivalis and at least two Prevotella spp. (Coyne et al., 2013). With respect to another major periodontal pathogen, Treponema denticola, a recent study points to the role of sialic acid modified surface glycoproteins in resisting the serum complement mediated killing of the pathogen (Kurniyati et al., 2013). However, basic information regarding the glycosylation machinery in this important pathogen is currently lacking. It is also worth noting that many species of Campylobacter associated with periodontitis (Macuch and Tanner, 2000), namely, $C$. rectus, C. showae, and $C$. concisus appear to also possess possible flagella glycosylation islands based on genome predictions (unpublished).

Furthermore, most intriguing is the presence of myriad glycosidases in the periodontal pathogen T. forsythia (Sharma, 2010), which otherwise depend on host proteinaceous components for growth. These include $\beta$-hexosaminidases (Roy et al., 2012) and most prominently sialidases (Braham and Moncla, 1992; Ishikura et al., 2003; Honma et al., 2011; Roy et al., 2011; Kurniyati et al., 2013) which appears to play a role in colonization and pathogenesis. We speculate that sialidases of periodontal bacteria could likely be involved in immune regulation as well, as is the case with the pneumococcal sialidase which can potentially induce leukocytes to release proinflammatory cytokines through relieving the dampening effect of Siglec receptors (Chang et al., 2012). Siglecs are sialic acid binding lectins on immune cells. Their activation by cognate sialoconjugates causes recruitment of phosphatases to the ITIM domain (immunoreceptor tyrosine-based inhibitory motif), leading to the inhibition of kinase-dependent activation of inflammatory signaling cascade in the cell (Crocker et al., 2007). Thus, hydrolysis of sialic acid from Siglec ligands by bacterial sialidases can potentially mitigate the immunosuppressive activity of Siglecs. Alternatively, sialidases may provide sialic acid to periodontal bacteria for incorporation into their cell-surface components, affording them the ability to undermine immune system in different ways. In $P$ gingivalis, a recent study demonstrated that bacterial sialidases play important roles in capsule formation and resistance to complement attack (Li et al., 2012).

\section{GLYCOMIMICRY}

Evading immune recognition is critical for pathogen survival in the host. Pathogens decorate their surfaces with molecular structures that can mimic host molecules to avoid detection by the immune system. For example, hyaluronic acid and $\alpha 2-8$-linked sialic acid polymers are abundant on capsule polysaccharides of group-A streptococci (GAS) and group-B streptococci (GBS). These structures resemble glycosaminoglycan and glycosylated proteins present on host connective, epithelial and neural tissues respectively. The GBS-expressed polysaccharide mimics a unique glycan structure found in human glycoproteins recognized by Siglecs, coopting of which by bacterial polysaccharide can lead to dampening of the immune responses including the impairment of neutrophil attack. Likewise, polysaccharides of Neisseria meningitidis and Escherichia coli $\mathrm{K} 1$ antigens are structurally similar to those of human fetal neuronal cells (Finne et al., 1987; Robbins et al., 2011). It was also demonstrated that this microbial glycomimicry can mislead the host immune system. For instance, carbohydrate mimicry between $C$. jejuni lipooligosaccharide and gangliosides has been implicated in the onset of paralytic disorder Guillain-Barré syndrome. In this setting, infection by $C$. jejuni in humans is believed to induce cross-reactive serum antibodies which bind to and damage peripheral nerves resulting in autoimmune disorders (Yuki et al., 2004). Interestingly, T. forsythia terminal sugar pseudaminic acid linked to an $O$-glycan core at least partially mimics sialic acid structures. Therefore, it is tempting to speculate that by abundantly decorating the surface with modified pseudaminic acid residues the bacterium could avoid immune surveillance and enhance its survival.

\section{CONCLUSION}

This review highlights the range and potential for immunomodulation by surface protein glycans in several periodontal bacteria. 
We also highlight the action of an exemplar glycan, namely that on the Tannerella S-layer that influences immune responses and promotes survival and persistence in the body while promoting bone loss orally. We hypothesize that bacterial glycans might have a prominent role in influencing local and systemic immunity to affect the persistence and pathogenicity of pathogenic oral communities in periodontal disease. Therefore, the future targeting of such

\section{REFERENCES}

Aruni, A. W., Robles, A., and Fletcher, H. M. (2013). VimA mediates multiple functions that control virulence in Porphyromonas gingivalis. Mol. Oral Microbiol. 28, 167-180. doi: 10.1111/omi.12017

Ashline, D., Singh, S., Hanneman, A., and Reinhold, V. (2005). Congruent strategies for carbohydrate sequencing. 1. Mining structural details by MSn. Anal. Chem. 77, 6250-6262. doi: $10.1021 /$ ac050724z

Avci, F. Y., and Kasper, D. L. (2010). How bacterial carbohydrates influence the adaptive immune system. Annu. Rev. Immunol. 28, 107-130. doi: 10.1146/annurev-immunol- 030409101159

Avci, F. Y., Li, X., Tsuji, M., and Kasper, D. L. (2013). Carbohydrates and T cells: a sweet twosome. Semin. Immunol. 25, 146-151. doi: 10.1016/j.smim.2013.05.005

Beck, J. D., Couper, D. J., Falkner, K. L., Graham, S. P., Grossi, S. G., Gunsolley, J. C., et al. (2008). The Periodontitis and Vascular Events (PAVE) pilot study: adverse events. J. Periodontol. 79, 90-96. doi: 10.1902/jop. 2008.070223

Braham, P. H., and Moncla, B. J. (1992). Rapid presumptive identification and further characterization of Bacteroides forsythus. J. Clin. Microbiol. 30, 649-654.

Brisson, J. R., Vinogradov, E., Mcnally, D. J., Khieu, N. H., Schoenhofen, I. C., Logan, S. M., et al. (2010). The application of NMR spectroscopy to functional glycomics. Methods Mol. Biol. 600, 155-173. doi: 10.1007/9781-60761-454-8 11

Chang, Y. C., Uchiyama, S., Varki, A., and Nizet, V. (2012). Leukocyte inflammatory responses provoked by pneumococcal sialidase. MBio 3, pii: e00220-e00211. doi: 10.1128/mBio.00220-11

Chauhan, J. S., Bhat, A. H., Raghava, G. P., and Rao, A. (2012). GlycoPP: a webserver for prediction of $N$ - and $\mathrm{O}$-glycosites in prokaryotic protein sequences. PLOS ONE 7:e40155. doi: 10.1371/journal.pone.0040155

Coyne, M. J., Fletcher, C. M., Chatzidaki-Livanis, M., Posch, G.,
Schaffer, C., and Comstock, L. E. (2013). Phylum-wide general protein $\mathrm{O}$-glycosylation system of the Bacteroidetes. Mol. Microbiol. 88 772-783. doi: $10.1111 / \mathrm{mmi} .12220$

Crocker, P. R., Paulson, J. C., and Varki, A. (2007). Siglecs and their roles in the immune system. Nat. Rev. Immunol. 7, 255-266. doi: 10.1038/nri2056

Dewhirst, F. E., Chen, T., Izard, J., Paster, B. J., Tanner, A. C., Yu, W. H., et al. (2010). The human oral microbiome. J. Bacteriol. 192, 5002-5017. doi: 10.1128/JB.00542-10

Eichler, J. (2013). Extreme sweetness: protein glycosylation in archaea. Nat Rev. Microbiol. 11, 151-156. doi: 10.1038/nrmicro2957

Finne, J., Bitter-Suermann, D., Goridis, C., and Finne, U. (1987). An IgG monoclonal antibody to group B meningococci cross-reacts with developmentally regulated polysialic acid units of glycoproteins in neural and extraneural tissues. J. Immunol. 138, 4402-4407.

Fletcher, C. M., Coyne, M. J., and Comstock, L. E. (2011). Theoretical and experimental characterization of the scope of protein $\mathrm{O}$-glycosylation in Bacteroides fragilis. J. Biol. Chem. 286, 3219-3226. doi: 10.1074/jbc.M110.194506

Fletcher, C. M., Coyne, M. J., Villa, O. F., Chatzidaki-Livanis, M., and Comstock, L. E. (2009). A general $O$ glycosylation system important to the physiology of a major human intestinal symbiont. Cell 137, 321-331. doi: 10.1016/j.cell.2009.02.041

Geijtenbeek, T. B., Den Dunnen, J., and Gringhuis, S. I. (2009). Pathogen recognition by DC-SIGN shapes adaptive immunity. Future Microbiol. 4, 879-890. doi: 10.2217/fmb. 09.51

Geijtenbeek, T. B., and Gringhuis, S. I. (2009). Signalling through C-type lectin receptors: shaping immune responses. Nat. Rev. Immunol. 9, 465-479. doi: 10.1038/nri2569

Genco, R. J. (2009). Periodontal disease and association with diabetes mellitus and diabetes: clinical implications. J. Dent. Hyg. 83, 186-187. doi:10.1902/annals.2001.6.1.99

interactions may provide a novel approach for modulating periodontitis.

\section{ACKNOWLEDGMENTS}

The work performed in authors' laboratories was supported by a grant (DE014749 to Ashu Sharma) from the National Institute of Dental and Craniofacial Research (Bethesda, MD) and by The Dunhill Medical Trust grant R185/0211 to Graham P. Stafford.

Genco, R. J., and Van Dyke, T. E. (2010). Prevention: reducing the risk of CVD in patients with periodontitis. Nat. Rev. Cardiol. 7, 479-480. doi: 10.1038/nrcardio. 2010.120

Glew, M. D., Veith, P. D., Peng, B., Chen, Y. Y., Gorasia, D. G., Yang, Q., et al. (2012). PG0026 is the C-terminal signal peptidase of a novel secretion system of Porphyromonas gingivalis. J. Biol. Chem. 287, 24605-24617. doi: 10.1074/jbc.M112.369223

Gomes-Filho, I. S., Santos, C. M., Cruz, S. S., Passos Jde, S., Cerqueira Ede, M., Costa Mda, C., etal. (2009). Periodontitis and nosocomial lower respiratory tract infection: preliminary findings. J. Clin. Periodontol. 36, 380-387. doi: 10.1111/j.1600051X.2009.01387.x

Gonzalez-Fernandez, A., Faro, J., and Fernandez, C. (2008). Immune responses to polysaccharides: lessons from humans and mice. Vaccine 26, 292-300. doi: 10.1016/j.vaccine.2007.11.042

Guerry, P., Ewing, C. P., Schirm, M., Lorenzo, M., Kelly, J., Pattarini, D., et al. (2006). Changes in flagellin glycosylation affect Campylobacter autoagglutination and virulence. Mol. Microbiol. 60, 299-311. doi $10.1111 / j .1365-2958.2006 .05100 . x$

Guo, Y., Nguyen, K. A., and Potempa, J. (2010). Dichotomy of gingipains action as virulence factors: from cleaving substrates with the precision of a surgeon's knife to a meat chopper-like brutal degradation of proteins. Periodontol. 2000 54, 15-44. doi: 10.1111/j.16000757.2010.00377.x

Hitchen, P., Brzostek, J., Panico, M., Butler, J. A., Morris, H. R., Dell, A., et al. (2010). Modification of the Campylobacter jejuni flagellin glycan by the product of the Cj1295 homopolymeric-tract-containing gene. Microbiology 156, 1953-1962. doi: 10.1099/mic.0.038091-0

Honma, K., Inagaki, S., Okuda, K., Kuramitsu, H. K., and Sharma, A. (2007). Role of a Tannerella forsythia exopolysaccharide synthesis operon in biofilm development. Microb. Pathog. 42, 156-166. doi 10.1016/j.micpath.2007.01.003
Honma, K., Mishima, E., and Sharma, A. (2011). Role of Tannerella forsythia $\mathrm{NanH}$ sialidase in epithelial cell attachment. Infect. Immun. 79, 393401. doi: 10.1128/IAI.00629-10

Ishikura, H., Arakawa, S., Nakajima, T., Tsuchida, N., and Ishikawa, I. (2003). Cloning of the Tannerella forsythensis (Bacteroidesforsythus) siaHI gene and purification of the sialidase enzyme. J. Med. Microbiol. 52, 1101-1107. doi: 10.1099/jmm.0.05349-0

Iwashkiw, J. A., Seper, A., Weber, B. S., Scott, N. E., Vinogradov, E., Stratilo, C., etal. (2012). Identification of a general $O$-linked protein glycosylation system in Acinetobacter baumannii and its role in virulence and biofilm formation. PLoS Pathog. 8:e1002758. doi: 10.1371/journal.ppat. 1002758

Iwashkiw, J. A., Vozza, N. F., Kinsella, R. L., and Feldman, M. F. (2013). Pour some sugar on it: the expanding world of bacterial protein $O$-linked glycosylation. Mol. Microbiol. 89, 14-28. doi: 10.1111/mmi.12265

Kandiba, L., and Eichler, J. (2013). Analysis of putative nonulosonic acid biosynthesis pathways in Archaea reveals a complex evolutionary history. FEMS Microbiol. Lett. 345, 110120. doi: 10.1111/1574-6968.12193

Karlyshev, A. V., Everest, P., Linton, D., Cawthraw, S., Newell, D. G., and Wren, B. W. (2004). The Campylobacter jejuni general glycosylation system is important for attachment to human epithelial cells and in the colonization of chicks. Microbiology 150, 1957-1964. doi: 10.1099/mic.0.26721-0

Kishi, M., Hasegawa, Y., Nagano, K., Nakamura, H., Murakami, Y., and Yoshimura, F. (2012). Identification and characterization of novel glycoproteins involved in growth and biofilm formation by Porphyromonas gingivalis. Mol. Oral Microbiol. 27, 458-470. doi: $10.1111 /$ j.20411014.2012.00659.x

Kuo, C., Takahashi, N., Swanson, A. F., Ozeki, Y., and Hakomori, S. (1996). An $N$-linked high-mannose type oligosaccharide, expressed at the major outer membrane protein of Chlamydia trachomatis, mediates 
attachment and infectivity of the microorganism to HeLa cells. J. Clin. Invest. 98, 2813-2818. doi: 10.1172/JCI119109

Kurniyati, K., Zhang, W., Zhang, K., and Li, C. (2013). A surfaceexposed neuraminidase affects complement resistance and virulence of the oral spirochete Treponema denticola. Mol. Microbiol. 89, 842-856. doi: 10.1111/mmi.12311

Lee, S. W., Sabet, M., Um, H. S., Yang, J., Kim, H. C., and Zhu, W. (2006). Identification and characterization of the genes encoding a unique surface (S-) layer of Tannerella forsythia. Gene 371, 102-111. doi: 10.1016/j.gene.2005.11.027

Li, C., Kurniyati, Hu, B., Bian, J., Sun, J., Zhang, W., et al. (2012). Abrogation of neuraminidase reduces biofilm formation, capsule biosynthesis, and virulence of Porphyromonas gingivalis. Infect. Immun. 80, 3-13. doi: 10.1128/IAI.05773-11

Macuch, P. J., and Tanner, A. C. (2000). Campylobacter species in health, gingivitis, and periodontitis. J. Dent. Res. 79, 785-792. doi: 10.1177/00220345000790021301

Maue, A. C., Mohawk, K. L., Giles, D. K., Poly, F., Ewing, C. P., Jiao, Y., et al. (2012). The polysaccharide capsule of Campylobacter jejuni 81-176 modulates the host immune response. Infect. Immun. 81, 665-672. doi: 10.1128/IAI.01008-12

Mazmanian, S. K., Round, J. L., and Kasper, D. L. (2008). A microbial symbiosis factor prevents intestinal inflammatory disease. Nature 453, 620-625. doi: 10.1038/nature07008

Meijerink, M., Ferrando, M. L., Lammers, G., Taverne, N., Smith, H. E., and Wells, J. M. (2012). Immunomodulatory effects of Streptococcus suis capsule type on human dendritic cell responses, phagocytosis and intracellular survival. PLoS ONE 7:e35849. doi: 10.1371/journal.pone.0035849

Messner, P., Steiner, K., Zarschler, K., and Schaffer, C. (2008). S-layer nanoglycobiology of bacteria. Carbohydr. Res. 343, 1934-1951. doi: 10.1016/j.carres.2007.12.025

Myneni, S. R., Settem, R. P., Connell, T. D., Keegan, A. D., Gaffen, S. L., and Sharma, A. (2011). TLR2 signaling and Th2 responses drive Tannerella forsythia-induced periodontal bone loss. J. Immunol. 187, 501-509. doi: 10.4049/jimmunol.110 0683

Myneni, S. R., Settem, R. P., Sojar, H. T., Malone, J. P., Loimaranta, V., Nakajima, T., et al. (2012). Identification of a unique TLR2-interacting peptide motif in a microbial leucinerich repeat protein. Biochem. Biophys. Res. Commun. 423, 577-582. doi: 10.1016/j.bbrc.2012.06.008

Nothaft, H., and Szymanski, C. M. (2010). Protein glycosylation in bacteria: sweeter than ever. Nat. Rev. Microbiol. 8, 765-778. doi: 10.1038/nrmicro2383

Ochoa-Reparaz, J., Mielcarz, D. W., Wang, Y., Begum-Haque, S., Dasgupta, S., Kasper, D. L., et al. (2010). A polysaccharide from the human commensal Bacteroides fragilis protects against CNS demyelinating disease. Mucosal Immunol. 3, 487-495. doi: 10.1038/mi.2010.29

Onishi, S., Honma, K., Liang, S., Stathopoulou, P., Kinane, D. Hajishengallis, G., et al. (2008). Toll-like receptor 2-mediated interleukin-8 expression in gingival epithelial cells by the Tannerella forsythia leucine-rich repeat protein BspA. Infect. Immun. 76, 198-205. doi: 10.1128/IAI.01139-07

Paramonov, N., Rangarajan, M. Hashim, A., Gallagher, A., AduseOpoku, J., Slaney, J. M., et al. (2005). Structural analysis of a novel anionic polysaccharide from Porphyromonas gingivalis strain W50 related to Arggingipain glycans. Mol. Microbiol. 58, 847-863. doi: 10.1111/j.13652958.2005.04871.x

Posch, G., Pabst, M., Brecker, L., Altmann, F., Messner, P., and Schaffer, C. (2011). Characterization and scope of S-layer protein $\mathrm{O}$ glycosylation in Tannerella forsythia. J. Biol. Chem. 286, 38714-38724. doi: 10.1074/jbc.M111.284893

Posch, G., Pabst, M., Neumann, L., Coyne, M. J., Altmann, F., Messner, P., et al. (2013). "Crossglycosylation" of proteins in Bacteroidales species. Glycobiology 23, 568-577. doi: 10.1093/glycob/cws172 Robbins, J. B., Schneerson, R., Xie, G., Hanson, L. A., and Miller, M. A. (2011). Capsular polysaccharide vaccine for Group B Neisseria meningitidis, Escherichia coli $\mathrm{K} 1$, and Pasteurella haemolytica A2. Proc. Natl. Acad. Sci. U.S.A. 108, 1787117875. doi: 10.1073/pnas.111448 9108

Roy, S., Honma, K., Douglas, I., Sharma, A., and Stafford, G. (2011). Role of sialidase in glycoprotein utilisation by Tannerella forsythia. Microbiology 157, 3195-3202. doi: 10.1099/mic.0.052 498-0

Roy, S., Phansopa, C., Stafford, P., Honma, K., Douglas, C. W., Sharma, A., et al. (2012). Betahexosaminidase activity of the oral pathogen Tannerella forsythia influences biofilm formation on glycoprotein substrates. FEMS Immunol. Med. Microbiol. 65, 116-120. doi: 10.1111/j.1574-695X.2012.00933.x

Sabet, M., Lee, S. W., Nauman, R. K., Sims, T., and Um, H. S. (2003) The surface (S-) layer is a virulence factor of Bacteroides forsythus. Microbiology 149, 3617-3627. doi: 10.1099/mic.0.26535-0

Sara, M., and Sleytr, U. B. (2000) S-Layer proteins. J. Bacteriol. 182, 859-868. doi: 10.1128/JB.182.4.859 868.2000

Scher, J. U., Ubeda, C., Equinda, M., Khanin, R., Buischi, Y., Viale, A., et al. (2012). Periodontal disease and the oral microbiota in newonset rheumatoid arthritis. Arthritis Rheum. 64, 3083-3094. doi: 10.1002/art.34539

Settem, R. P., Honma, K., Nakajima, T., Phansopa, C., Roy, S., Stafford, G. P., et al. (2012). A bacterial glycan core linked to surface (S)-layer proteins modulates host immunity through Th17 suppression. Mucosal Immunol. 6, 415-426. doi: 10.1038/mi.2012.85

Sharma, A. (2010). Virulence mechanisms of Tannerella forsythia. Periodontol. 2000 54, 106-116. doi: 10.1111/j.1600-0757.2009.00332.x

Socransky, S. S., Haffajee, A. D., Cugini, M. A., Smith, C., and Kent, R. L. Jr. (1998). Microbial complexes in subgingival plaque. J. Clin. Periodontol. 25, 134-144. doi: 10.1111/j.1600051X.1998.tb02419.x

Surana, N. K., and Kasper, D. L. (2012). The yin yang of bacterial polysaccharides: lessons learned from B. fragilis PSA. Immunol. Rev. 245, 13-26. doi 10.1111/j.1600-065X.2011.01075.x

Szymanski, C. M., Burr, D. H., and Guerry, P. (2002). Campylobacter protein glycosylation affects host cell interactions. Infect. Immun. 70, 2242-2244. doi: 10.1128/IAI.70.4.2242-2244.2002

Szymanski, C. M., and Wren, B. W. (2005). Protein glycosylation in bacterial mucosal pathogens. Nat. Rev. Microbiol. 3, 225-237. doi: 10.1038/nrmicro1 100

Takeuchi, O., and Akira, S. (2010). Pattern recognition receptors and inflammation. Cell 140, 805-820. doi: 10.1016/j.cell.2010.01.022

Tzianabos, A. O., Finberg, R. W., Wang, Y., Chan, M., Onderdonk, A. B., Jennings, H. J., et al. (2000). T cells activated by zwitterionic molecules prevent abscesses induced by pathogenic bacteria. J. Biol. Chem. 275, 67336740. doi: 10.1074/jbc.275.10.6733

van den Berg, L. M., Gringhuis, S. I., and Geijtenbeek, T. B.
(2012). An evolutionary perspective on C-type lectins in infection and immunity. Ann. N. Y. Acad. Sci. 1253, 149-158. doi: 10.1111/j.17496632.2011.06392.x

Vik, A., Aas, F. E., Anonsen, J. H., Bilsborough, S., Schneider, A., EggeJacobsen, W., et al. (2009). Broad spectrum $\mathrm{O}$-linked protein glycosylation in the human pathogen Neisseria gonorrhoeae. Proc. Natl. Acad. Sci. U.S.A. 106, 4447-4452. doi: 10.1073/pnas.0809504106

Young, K. T., Davis, L. M., and Dirita, V. J. (2007). Campylobacter jejuni: molecular biology and pathogenesis. Nat. Rev. Microbiol. 5, 665-679. doi: 10.1038/nrmicro1718

Yuki, N., Susuki, K., Koga, M., Nishimoto, Y., Odaka, M., Hirata, K., et al. (2004). Carbohydrate mimicry between human ganglioside GM1 and Campylobacter jejuni lipooligosaccharide causes GuillainBarré syndrome. Proc. Natl. Acad. Sci. U.S.A. 101, 11404-11409. doi: 10.1073/pnas.0402391101

Zeituni, A. E., Mccaig, W., Scisci, E., Thanassi, D. G., and Cutler, C. W. (2010). The native 67kilodalton minor fimbria of Porphyromonas gingivalis is a novel glycoprotein with DC-SIGN-targeting motifs. J. Bacteriol. 192, 4103-4110. doi: 10.1128/JB.00275-10

Conflict of Interest Statement: The authors declare that the research was conducted in the absence of any commercial or financial relationships that could be construed as a potential conflict of interest.

Received: 14 August 2013; accepted: 27 September 2013; published online: 17 October 2013.

Citation: Settem RP, Honma K, Stafford GP and Sharma A (2013) Protein-linked glycans in periodontal bacteria: prevalence and role at the immune interface. Front. Microbiol. 4:310. doi: 10.3389/ fmicb.2013.00310

This article was submitted to Microbial Immunology, a section of the journal Frontiers in Microbiology.

Copyright (C) 2013 Settem, Honma, Stafford and Sharma. This is an openaccess article distributed under the terms of the Creative Commons Attribution License (CC BY). The use, distribution or reproduction in other forums is permitted, provided the original author(s) or licensor are credited and that the original publication in this journal is cited, in accordance with accepted academic practice. No use, distribution or reproduction is permitted which does not comply with these terms. 